In 2004, after long and controversial consultations, study leaders adopted a household-based sampling strategy, a costly and labour-intensive decision meaning that participants from the 105 far-flung study locations will be more representative of the broad population than, say, those recruited from health facilities and doctor's offices, a more common approach.

In May 2008, in a 140-page report, the National Research Council and the Institute of Medicine praised the study's householdbased sampling approach, as well as the statistical power rendered by its 100,000 participants. But it also identified a laundry list of weaknesses in the study's design. The first was the absence of a pilot phase; because the vanguard centres' data were to be part of the final data set, investigators would be less likely to experiment with methods and change things that weren't working. That concern is being addressed, says Shurin, by turning the vanguard centres into longterm development platforms for the main study's methodology and feasibility, and not, as initially intended, folding them into the main study. Study leaders have also pushed back the launch of the study's main phase until late 2011, giving investigators time to tweak the methodology according to what's learned at the vanguard centres.

Despite the rough waters, advocates remain passionate about the study's potential, arguing that it is an investment that will more than pay for itself. Leonardo Trasande, a study investigator at the Mount Sinai School of Medicine in New York, notes that just six of the conditions the study explores - asthma, autism, diabetes, injury, obesity and schizophrenia - cost the United States at least $\$ 650$ billion a year (L. Trasande and P. J. Landrigan Environ. Health Persp. 112, A789-A790; 2004). "If the study identifies preventive interventions that can reduce those annual costs by even $1 \%$, [a $\$ 3$-billion] study repays itself twofold in one year," he says.

Meanwhile, Duplin County continues to enrol participants. While recruiters work church dinners and organize soccer tournaments, data collectors recently finished enumerating 10,800 households - roughly half of those in the county - aiming to locate reproductive-age women.

"I have two daughters. Both have asthma. I have lived this," says Entwisle. "But the scientific reason for my involvement has to do with the fact that there are so many questions that need answers. This design is exactly what needs to be done to begin to develop some of those answers."

Meredith Wadman

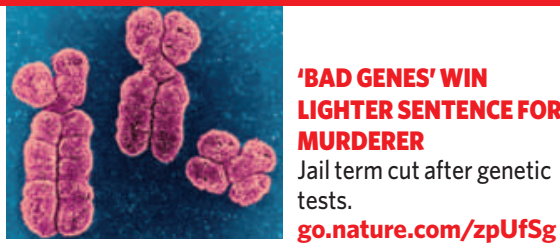

\title{
10,000 genomes to come
}

Results are just beginning to arrive for the 1000 Genomes Project, a genomic study of human diversity. However, an international group is already planning something even more ambitious - a 10,000 genomes project. The initiative, called Genome $10 \mathrm{~K}$, aims to tackle thousands of vertebrate species.

Project members write this week in the Journal of Heredity that the effort will provide an unprecedented look at the genomic mechanisms for generating diversity in a group of animals with different lifestyles and adaptations.

"We see this stunning diversity of forms in vertebrates, from manatees to anteaters,"

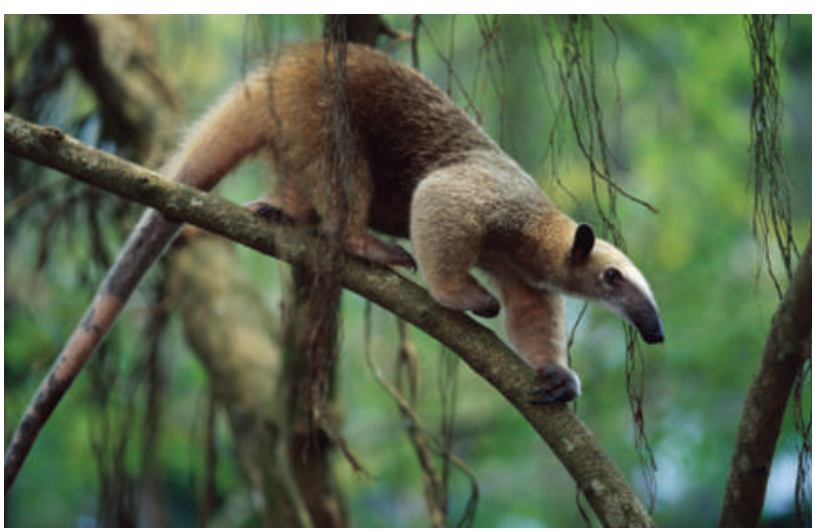

The anteater could have its genome sequenced.

says David Haussler of the University of California, Santa Cruz, one of the three masterminds behind the project, which involves 68 scientists from five continents. "What continually strikes me is the unbelievable malleability and adaptability of the vertebrate genome," he says, "and we have an enormous amount to learn about the genetic roots of that."

Haussler, Stephen O'Brien of the National Cancer Institute in Frederick, Maryland, and Oliver Ryder, director of genetics at the San Diego Zoo's Institute for Conservation Research in Escondido, California, decided to organize the project after realizing that one of the major obstacles to non-human sequencing projects has been collecting and organizing specimens. After an April meeting in Santa Cruz to bring in other scientists, the team now has a database of samples from more than 16,000 species from 50 institutions. The scientists are also planning a pilot project to sample portions of the genomes of a small subset of these species.
The group is looking for funding for the main phase of the project, which could cost anywhere from US\$10 million to $\$ 100$ million, depending on the costs to process and sequence each sample. The team anticipates that sequencing costs will drop below $\$ 10,000$ per genome within a few years, making it feasible to sequence the entire genomes of 10,000 vertebrates within this budget.

However, analysing all the data will be an enormous challenge, because it is still not easy to assemble new genomes from the short readouts of DNA delivered by current sequencing technologies.

Other scientists say that the project sounds exciting, but question the decision to sequence so deeply solely among vertebrates.

David Maddison, who studies beetle phylogeny at Oregon State University in Corvallis, points out that, so far, only one beetle species has had its genome sequenced, despite there being about six times as many beetle species as there are vertebrates.

"My biggest concern is that if one were to decide where our funds should go - to 10,000 vertebrates or more generally scattered across organismal diversity - I would very strongly argue for the latter," says Maddison, who conceived the Tree of Life project, a web-based catalogue of biodiversity.

O'Brien agrees that invertebrate-genome sequencing is "a valuable area that should also be considered for whole-genomesequence assessment". Other scientists are already planning a large invertebrate sequencing project.

Indeed, O'Brien expects Genome $10 \mathrm{~K}$ to be the first study in what he anticipates will be a larger shift toward using sequencing technology to study biodiversity. He compares it to the culture shift that accompanied the invention of the printing press, which was first used to print the Bible and then for broader purposes.

"If the Human Genome Project is the Bible," he says, "then the [Genome $10 \mathrm{~K}$ ] is a library that gets filled up with other books."

Erika Check Hayden 